\title{
The Role of Purkinje Automaticity as an Arrhythmia Mechanism in Hyperkalaemia
}

\author{
Violeta Monasterio $^{1}$, Jesús Carro ${ }^{1,2}$, Esther Pueyo ${ }^{2,3}$, José F Rodríguez ${ }^{2,4}$ \\ ${ }^{1}$ Universidad San Jorge, Villanueva de Gállego, Spain \\ ${ }^{2}$ Universidad de Zaragoza, Zaragoza, Spain \\ ${ }^{3}$ CIBER-BBN, Zaragoza, Spain \\ ${ }^{4}$ Politecnico di Milano, Milano, Italy
}

\begin{abstract}
The pacemaker activity of Purkinje (PK) cells is usually suppressed by the sino-atrial (SA) node. Under abnormal conditions, PK cells may produce ectopic focal activity leading to tachycardia and fibrillation. This computational modelling work illustrates the potential role of $P K$ automaticity as an arrhythmia mechanism in hyperkalaemia.

First, we investigated the automaticity of isolated PK cells under hyperkalaemic conditions. Second, the potential consequences of altered $P K$ automaticity were explored in a 1-D model of a terminal PK fibre running through a hyperkalaemic zone. Hyperkalaemia was found to accelerate the autorhythmic period of isolated PK cells with respect to control. For extracellular potassium levels $\geq 7.2 \mathrm{mM}$, spontaneous action potentials (APs) appeared in the PK fibre, leading to conduction disturbances and complex combinations of spontaneous and stimulationdriven APs.
\end{abstract}

\section{Introduction}

Under normal conditions, the SA node is the prevailing pacemaker of the heart. Abnormal automaticity occurs when other cells, like cells in the atrio-ventricular node or in the His-Purkinje conduction system, undertake the SA node function [1]. Under pathological conditions, the SA node cells may reduce their rate of spontaneous depolarization. Even in the case of normal SA node functioning, some cells can develop rates of firing faster than that of the SA node. In particular, both reentrant-triggered and enhanced automatic rhythms can arise from the Purkinje (PK) fibre network, leading to ventricular arrhythmias [2].

In this work we study the influence of hyperkalaemia (that is, an increase in the extracellular potassium concentration $\left[K^{+}\right]_{o}$ ) in PK cells activity. Hyperkalaemia is one of the main physiological changes that occur in cardiac cells during acute ischaemia [3], and has been confirmed to be an important factor in the genesis of reentrant-triggered rhythms at the PK-myocardium interface, since it regulates the range of intercellular coupling in which unidirectional conduction blocks may be induced [4].

In the present work we investigate the potential role of PK automaticity as an additional arrhythmia mechanism. For our computational simulation study we use two human action potential (AP) models: the ten Tusscher and Panfilov 2006 (TT06) endocardial model [5] and the Stewart et al. 2009 (ST09) model [6]. We start by studying the alterations in isolated PK cells automaticity under hyperkalaemia, and then we investigate the effects that those alterations have on the genesis of abnormal rhythms in a 1-D model of a PK fibre running towards the centre of an acutely hyperkalaemic endocardial zone.

\section{Methods}

First, the automaticity of isolated PK cells under hyperkalaemic conditions was investigated. Second, the effects of PK automaticity under hyperkalaemic conditions were explored in a 1-D model of the PK-ventricle subsystem. All simulations were carried out using the software ELVIRA [7].

\subsection{Isolated Purkinje cells}

The system of differential equations that govern the cellular electrical behaviour according to the ST09 model [6] was solved by using forward Euler integration with a time step $\Delta t=0.02 \mathrm{~ms}$. Transmembrane potential $\left(V_{m}\right)$ and all the other state variables were computed at each time step.

Hyperkalaemic conditions were simulated by increasing $\left[K^{+}\right]_{o}$ from 5.4 to $12.5 \mathrm{mM}$ with $0.1-\mathrm{mM}$ steps. For each $\left[K^{+}\right]_{o}$, the model was run without stimulation for $150 \mathrm{~s}$, and the average cycle length (CL) of spontaneous APs between $t=50 \mathrm{~s}$ and $t=150 \mathrm{~s}$ was measured. 


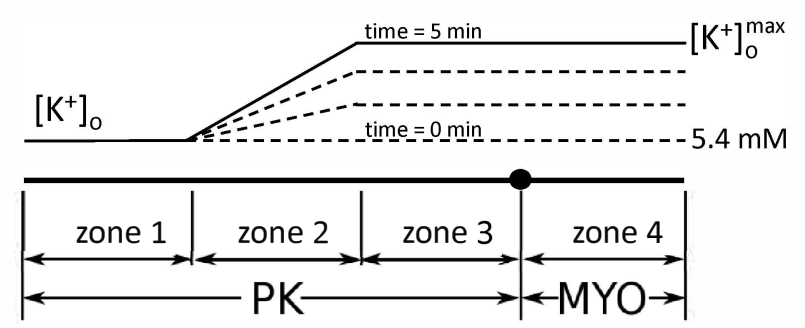

Figure 1. Purkinje-ventricle 1-D simulation setup.

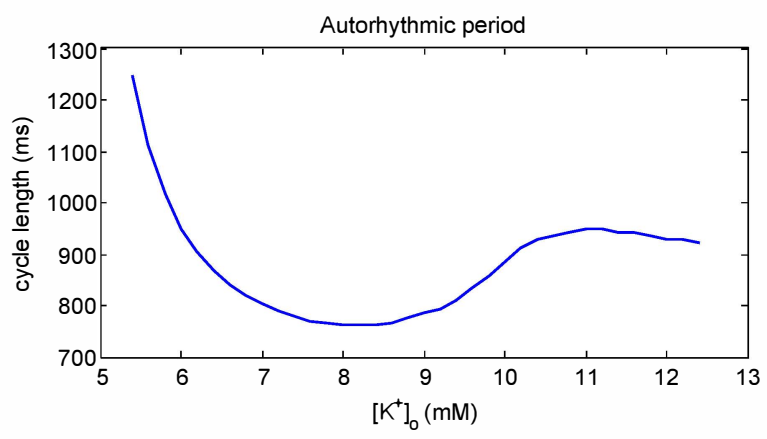

Figure 2. Average cycle length (CL) of spontaneous action potentials (APs) vs. $\left[K^{+}\right]_{o}$ in isolated PK cells.

\subsection{1-D Purkinje-ventricle tissue}

The PK-ventricle subsystem was modelled as a 1-D tissue composed of $3 \mathrm{~cm}$ of PK cells (zones 1 to 3 in Figure 1) connected to $1 \mathrm{~cm}$ of endocardial cells (TT06, zone 4 in Figure 1). Electric propagation across the cardiac tissue was modelled with the monodomain model. Discretization was performed using a spatial interval of $\Delta x=0.1 \mathrm{~mm}$ and a time interval of $\Delta t=0.02 \mathrm{~ms}$. Longitudinal conductivity was set to $\sigma_{p}=0.0068 \mathrm{mS} / \mathrm{cm}$ in zones 1 to 3 and to $\sigma_{m}=$ $0.0009 \mathrm{mS} / \mathrm{cm}$ in zone 4 , leading to conduction velocities of $2.1 \mathrm{~m} / \mathrm{s}$ and $0.7 \mathrm{~m} / \mathrm{s}$ respectively under control conditions, well within experimental ranges [8]. The Purkinjemyocardial junction was modelled as a resistor with $\sigma_{p}=$ $0.01 \mathrm{mS} / \mathrm{cm}$. The system of equations was solved using the software ELVIRA [7]. Twelve positions within the fibre were selected for further analysis in each simulation; those positions were located at $x_{i}-0.5, x_{i}$, and $x_{i}+0.5$ $\mathrm{mm}$, with $x_{i}$ being the central position in zone $i$.

The fibre was stimulated at the PK end, with current pulses of 1-ms duration and 1000-nA/pF amplitude, at CL $=1000 \mathrm{~ms}$ for $5 \mathrm{~min}$. During that time, $\left[K^{+}\right]_{O}$ was uniformly increased from $5.4 \mathrm{mM}$ to $\left[K^{+}\right]_{o}^{\max }$ in zones 3 and 4 , whereas $\left[K^{+}\right]_{O}$ remained at $5.4 \mathrm{mM}$ in zone 1 ; in zone $2,\left[K^{+}\right]_{o}$ varied linearly between the values in zones 1 and 3 (Figure 1). Simulations were carried out for $\left[K^{+}\right]_{o}^{\max }$ of $5.4,6.3,7.2$ and $8.1 \mathrm{mM}$.

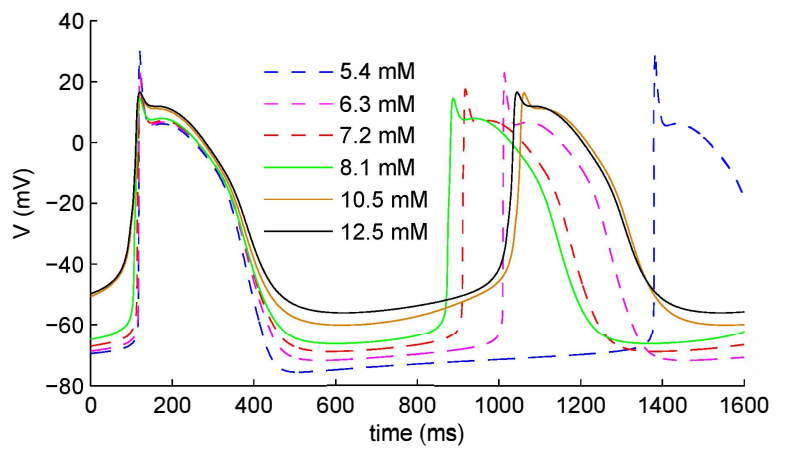

Figure 3. Spontaneous APs vs. $\left[K^{+}\right]_{O}$ in isolated PK cells. Traces were extracted from the last two seconds of each simulation and aligned for illustration purposes.

\section{Results}

\subsection{Isolated Purkinje cells}

The autorhythmic period of PK cells was strongly influenced by hyperkalaemia, with average CLs decreasing from $1248 \mathrm{~ms}\left(\left[K^{+}\right]_{O}=5.4 \mathrm{mM}\right)$ to a minimum of 762 $\mathrm{ms}\left(\left[K^{+}\right]_{o}=8.1 \mathrm{mM}\right)$ (Figure 2). AP-duration (APD) and resting potential (RP) were also affected by $\left[K^{+}\right]_{O}$. Figure 3 illustrates these results; APD increased from $310\left(\left[K^{+}\right]_{O}\right.$ $=5.4 \mathrm{mM})$ to $344\left(\left[K^{+}\right]_{o}=12.5 \mathrm{mM}\right)$, and the most negative RP varied from $-75.72 \mathrm{mV}\left(\left[K^{+}\right]_{o}=5.4 \mathrm{mM}\right)$ to $56.02\left(\left[K^{+}\right]_{o}=12.5 \mathrm{mM}\right)$.

\subsection{1-D Purkinje-ventricle tissue}

In the PK-ventricle model, no automaticity was observed in PK cells for $\left[K^{+}\right]_{o}^{\max }=5.4$ and $6.3 \mathrm{mM}$. In those cases, stimulation-generated APs propagated from the PK end of the fibre towards the myocardium end without disturbances. Figure 4(a) illustrates this situation. APs are generated at the PK end every $1000 \mathrm{~ms}$. The positive time delays between successive zones and the blue-red-black order in the zoom traces clearly indicate normal propagation towards the myocardium.

For $\left[K^{+}\right]_{o}^{\max }=7.2$ and $8.1 \mathrm{mM}, \mathrm{PK}$ automaticity was observed in the hyperkalaemic portion of the fibre (zone 3 ), which led to complex patterns of spontaneous and stimulation-driven APs by the end of the 5-min simulation. The time-course of this phenomenon was similar in both cases. Spontaneous APs appeared in zone 3 at $t=$ $226 \mathrm{~s}$ for $\left[K^{+}\right]_{o}^{\max }=7.2 \mathrm{mM}$ and at $t=156 \mathrm{~s}$ for $\left[K^{+}\right]_{o}^{\max }$ $=8.1 \mathrm{mM}$. A few seconds later, spontaneous APs started to propagate backwards (towards zone 2). By $t=250 \mathrm{~s}$ $\left(\left[K^{+}\right]_{o}^{\max }=7.2 \mathrm{mM}\right)$ and $t=176 \mathrm{~s}\left(\left[K^{+}\right]_{o}^{\max }=8.1 \mathrm{mM}\right)$ spontaneous APs reached the PK end of the fibre, creating mixed patterns of spontaneous and stimulation-driven 
APs. An example is shown in Figure 4(b), where spontaneous APs have overridden stimulated APs due to their shorter CL. A little time after $t=298.6 \mathrm{~ms}$, an spontaneous AP starts in zone 3 and propagates backwards (there is a positive time delay from zone 3 to zones 2 and 1, and within zones 2 and 1 the black-red-blue order of the traces indicate retrograde conduction), while a stimulation-driven AP is generated at the PK end at $t=298.6 \mathrm{~ms}$ and travels forward (towards the myocardium).

\section{Discussion and conclusions}

In this study we evaluated the effects of hyperkalaemia on PK automaticity. According to our results, mildly hyperkalaemic conditions may accelerate the rate of spontaneous discharge, while severe hyperkalaemic conditions seem to invert that tendency. This behaviour is consistent with some well-known effects of hyperkalaemia on cardiac cells [9]. An effect of mild hyperkalaemia is to shift the resting membrane potential to a less negative value. Although the threshold potential also becomes less negative, the magnitude of that change is smaller than the change in resting potential, so the net effect is to bring the resting potential closer to threshold potential, increasing cell excitability. On the other hand, the resting potential determines the number of voltage-gated sodium channels available for depolarization, which in turn determines the magnitude of the inward sodium current at the onset of phase 0 and thus the AP amplitude. As extracellular potassium levels increase, the resting membrane potential becomes less negative, reaching a point where the number of sodium channels available for depolarization severely decreases. The changes in threshold potential now are comparable to the changes in resting potential, so the cell becomes less excitable than in mild hyperkalaemia. These differences in excitability together with the progressive diastolic depolarization of PK cells may account for the differences in the autorhythmic period found in our study.

Our results on isolated $\mathrm{PK}$ cells showed changes in AP amplitude, RP and upstroke velocity that are consistent with the hypothesis above, and also with in vitro studies with human PK cells [10]. As a limitation, however, it should be noted that the changes found in APD are inconsistent with the literature $[10,11]$, since a shortening of APD has been reported under hyperkalaemia. To investigate whether the mechanisms behind this disagreement could also be affecting the automaticity rate, we performed a sensitivity analysis by varying the conductances and time constants of all currents in the ST09 model within $\pm 30 \%$ of their default value, but we did not observe any direct relationship between changes in automaticity rate (mainly determined by $I_{f}$ current) and the slight increase of APD. This observation, together with the fact that other human PK models also predict automaticity acceleration while correctly reflecting APD shortening [12], suggest that it is unlikely that the two phenomena are coupled.

Hyperkalaemia has been found to potentiate conduction abnormalities in clinical studies, in which abnormal impulse formation has been observed as a result of acceleration of normal pacemakers or the emergence of ectopic pacemakers [13]. Unfortunately, experimental observations on the effects of hyperkalaemia on the automaticity of human PK cells are scarce and mostly anecdotal. Although results in this work can only be considered as preliminar, the abnormal rhythms and complex patterns found in the PK-ventricle simulations suggest that altered PK automaticity under hyperkalaemia may play an important role in the generation of ventricular arrhythmias.

\section{Acknowledgements}

This work was supported by Ministerio de Economía y Competitividad (Spain) and FEDER (EU), under projects TIN2012-37546-C0303 and TIN2013-41998-R, by CIBER de Bioingeniería, Biomateriales y Nanomedicina (CIBER-BBN) through Instituto de Salud Carlos III and by Grupos Consolidados BSICoS (T96) and AMB (T88), from DGA and European Social Fund. E.P. acknowledges financial support of 'Ramón y Cajal' program from Ministerio de Economía y Competitividad (Spain).

\section{References}

[1] Laguna P, Martínez JP, Pueyo E. Techniques for cardiac instability assessment from the ECG. Proceedings of the IEEE 2015; In press.

[2] Boyden $\mathrm{Pa}$, Hirose M, Dun W. Cardiac Purkinje cells. Heart Rhythm 2010;7(1):127-135.

[3] Carmeliet E. Cardiac ionic currents and acute ischemia: from channels to arrhythmias. Physiological reviews 1999; 79(3):917-1017.

[4] Ramirez E, Saiz J, Romero L, Ferrero JM, Trenor B. In silico ischaemia-induced reentry at the Purkinje-ventricle interface. Europace 2014;16(3):444-451.

[5] ten Tusscher KHWJ, Panfilov AV. Alternans and spiral breakup in a human ventricular tissue model. Am J Physiol Heart Circ 2006;291(3):H1088-H1100.

[6] Stewart P, Aslanidi OV, Noble D, Noble PJ, Boyett MR, Zhang H. Mathematical models of the electrical action potential of Purkinje fibre cells. Philos Trans R Soc Lond A 2009;367(1896):2225-2255.

[7] Heidenreich EA, Ferrero JM, Doblaré M, Rodríguez JF. Adaptive macro finite elements for the numerical solution of monodomain equations in cardiac electrophysiology. Annals of biomedical engineering 2010;38(7):2331-2345.

[8] Katz AM. Physiology of the Heart. Lippincott Williams \& Wilkins, 2010.

[9] Parham Wa, Mehdirad Aa, Biermann KM, Fredman CS. Hyperkalemia revisited. Texas Heart Institute journal from the Texas Heart Institute of St Lukes Episcopal Hospital Texas Childrens Hospital 2006;33(1):40-47.

[10] Christé G. Effects of low [K+]o on the electrical activity of human cardiac ventricular and Purkinje cells. Cardiovascular research 1983;17(4):243-250. 

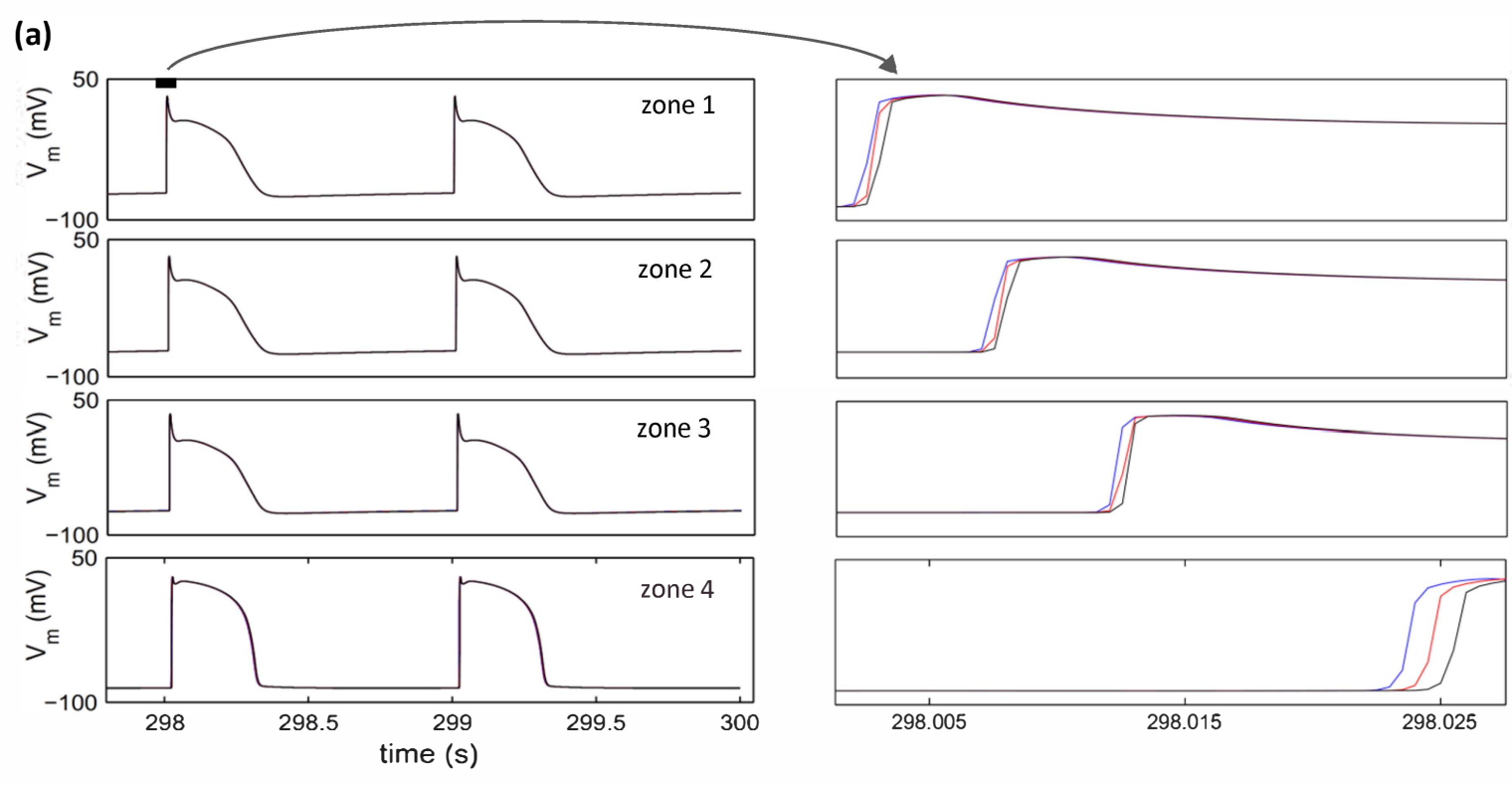

(b)

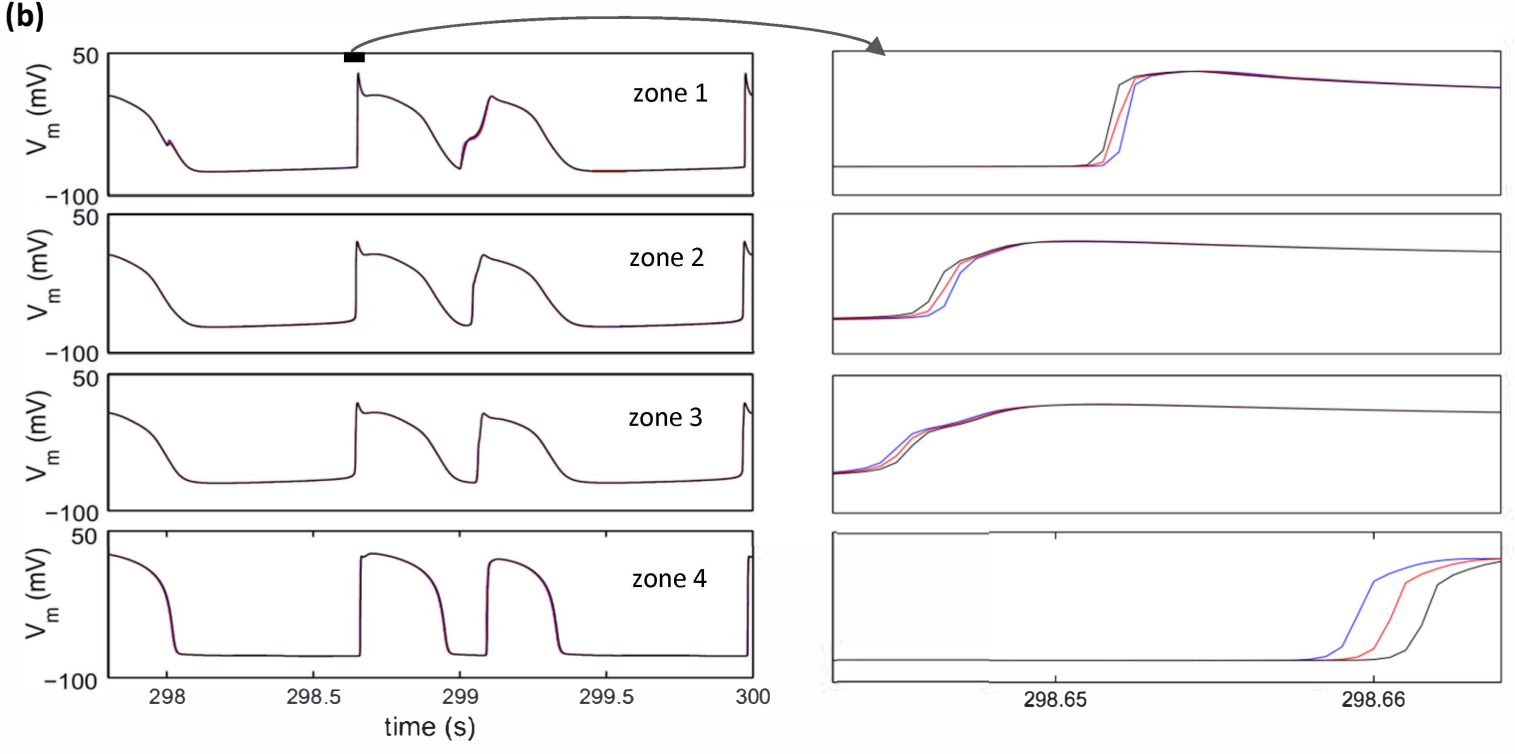

Figure 4. (a) 1-D simulation for $\left[K^{+}\right]_{o}^{\max }=5.4 \mathrm{mM}$. Top to bottom panels represent zones 1 to 4 as defined in the text. The last two seconds of simulation are shown on the left; a zoom is shown on the right. Membrane potentials at positions $x_{i}-0.5, x_{i}$, and $x_{i}+0.5 \mathrm{~mm}$, with $x_{i}$ being the central position in zone $i$, are shown in blue, red and black respectively. (b) Same representation for $\left[K^{+}\right]_{o}^{\max }=7.2 \mathrm{mM}$ results.

[11] Vassalle M. Cardiac pacemaker potentials at different extraand intracellular $\mathrm{K}$ concentrations. American Journal of Physiology Legacy Content 1965;208(4):770-775.

[12] Sampson KJ, Iyer V, Marks aR, Kass RS. A computational model of Purkinje fibre single cell electrophysiology: implications for the long QT syndrome. The Journal of physiology 2010;588(Pt 14):2643-2655.

[13] Cohen HC, Rosen KM, Pick A. Disorders of impulse con- duction and impulse formation caused by hyperkalemia in man. American heart journal 1975;89(4):501-509.

Address for correspondence:

Violeta Monasterio (vmonasterio@usj.es) Universidad San Jorge

50830 Villanueva de Gállego, Spain 